\title{
Flusskostenrechnung versus Umweltkennzahlen
}

\author{
Ein gutes Umweltmanagement bedarf einer aussagekräftigen Informations- \\ grundlage. Hierfür wurden in den letzten 20 Jahren zahlreiche Instrumente \\ entwickelt. Der Klassiker für die Optimierung der Stoff- und Energieströme \\ sind betriebliche Umweltbilanzen. Diese stoßen jedoch an Leistungsgrenzen. \\ Mit der Flusskostenrechnung und Umweltkennzahlen stehen zwei neuere \\ Instrumente zur Verfügung, die eine systematischere Analyse erlauben. \\ Für welches davon sollte sich ein Unternehmen entscheiden?
}

I

Von Thomas Loew und Gunnar Jürgens

nstrumente des Umweltmanagements erfuillen sehr unterschiedliche Funktionen, die beispielsweise von der Feststellung der betrieblichen Umweltwirkungen bis zur ökologischen Analyse des Produktlebenszyklusses reichen. Die meisten stehen daher nicht in Konkurrenz zueinander, sondern ergänzen sich gegenseitig. Für die öko-effiziente Optimierung der betrieblichen Stoff- und Energieströme wurden bereits Mitte der 80-er Jahre die betrieblichen Umweltbilanzen entwickelt. Ursprünglich waren nach diesem Ansatz nicht nur Bilanzen vorgesehen, die das Unternehmen bzw. einen Standort als „black box" betrachten, sondern auch Prozessbilanzen, die die Stoffströme einzelner Prozesse abbilden. Während sich diese Prozessbilanzen aufgrund des hohen Arbeitsaufwands bei der Erstellung nicht durchsetzen konnten, gehören die betrieblichen Umweltbilanzen in Unternehmen mit Umweltmanagementsystemen zu den Standardinstrumenten. Auch wenn die EG-Öko-Audit-Verordnung Umweltbilanzen nicht explizit verlangt, haben die meisten validierten Standorte im Rahmen der ersten Umweltprïfung eine Umweltbilanz erstellt.

Die betrieblichen Umweltbilanzen jedoch geben nur erste Anhaltspunkte auf der Suche nach ökologisch vorteilhaften Optimierungspotenzialen, indem sie z.B. auf hohe Verbräuche oder hohe Schadstoffmengen aufmerksam machen und somit die Suche nach Verbesserungsmöglichkeiten in einzelnen Prozessen und Bereichen anstoßen. Für die systematische Analyse der betrieblichen Stoff- und Energieströme sind sie jedoch zu grob. Hierfuir stehen inzwischen zwei
Instrumente, Umweltkennzahlen und die Flusskostenrechnung, zur Verfügung, die bereits in mehreren Unternehmen mit Erfolg eingesetzt werden. In welchem Verhältnis stehen diese beiden Informationsinstrumente zueinander, und welches Instrument kann am besten zur Verbesserung der betrieblichen Umweltleistung beitragen?

\section{Umweltkennzahlen}

Bereits 1990 wurden, begleitet von verschiedenen Forschungsprojekten, die Arbeiten für die Entwicklung einer ISO Norm zur Anwendung von Umweltkennzahlen aufgenommen. Die daraus entstandene ISO 14031 „Umweltmanagement Umweltleistungsbewertung - Leitlinien" wird noch 1999 als internationale Norm verabschiedet. Von den in der Norm beschriebenen Kennzahlen sind für das betriebliche Umweltmanagement zwei Arten von besonderer Bedeutung: Operative Leistungskennzahlen, die die Entwicklung der betrieblichen Stoffströme beschreiben und Managementleistungskennzahlen, die den Zustand und die Maßnahmen des Umweltmanagementsystems darstellen (1). Für den hier vorgenommenen Instrumentenvergleich werden vorwiegend die operativen Leistungskennzahlen betrachtet. Diese Leistungskennzahlen können verschiedene Funktionen erfüllen:

- Darstellung von Trendentwicklungen: Je nach Sachverhalt können absolute und/oder relative Periodenwerte Trendentwicklungen verdeutlichen.

- Erkennung von Schwachstellen: Kennzahlen können zur Untersuchung von Prozessen auf Optimierungspotenziale eingesetzt werden. Die Schwachstellen lassen sich über Zeitvergleiche oder Vergleiche zwischen gleichartigen Prozessen erkennen. So wurde beispielsweise anhand von Kennzahlen in der Kunststoffspritzerei von Hans Grohe erkannt, dass bedingt durch die geringere Kapazitätsauslastung in der Urlaubszeit für die Herstellung eines einzelnen Duschkopfs erheblich höhere Energiemengen aufgewendet wurden.

- Zielsetzung und Zielverfolgung im kontinuierlichen Verbesserungsprozess (KVP): Zum Teil können bestimmte Verbräuche oder Emissionen unmittelbar durch das Verhalten einzelner Mitarbeiter oder ganzer Teams beeinflusst werden. Mit Hilfe von entsprechenden Umweltkennzahlen können Aushänge und Berichte gestaltet werden, die zeitnah über die Entwicklung der betrachteten Stofflüsse informieren. Mit diesen Kennzahlen lassen sich dann Zielvorgaben formulieren und anschließend deren Einhaltung überprüfen.

Umweltkennzahlensysteme bilden Ausschnitte der betrieblichen Stofflüsse ab. Die Wahl der abgebildeten Ausschnitte hängt sowohl von der Beeinflussbarkeit der Stoffflüsse durch einzelne Mitarbeiter als auch von der Datenlage ab. Es kommt regelmäßig vor, dass bestimmte Kennzahlen prinzipiell gewünscht werden, aber die erforderlichen Daten zur Bildung der Kennzahlen noch nicht vorliegen.

\section{Flusskostenrechnung}

Die Flusskostenrechnung wird gemeinsam mit der Umweltschutzkostenrechnung und der für die betriebliche Praxis wenig relevanten Monetarisierung zu den Ansätzen der Umweltkostenrechnung gezählt. Die Unternehmen sind aufgrund des deutschen Umweltstatistikgesetzes inzwischen gezwungen, ihre Umweltschutzkosten, also die Investitionen, Abschreibungen und laufende Betriebskosten für Umweltschutzanlagen und für die externe Entsorgung, zu berechnen. Daher ist dieser Ansatz in unterschiedlichen Ausprägungen inzwischen vergleichsweise weit verbreitet.

Allmählich setzt sich jedoch die Erkenntnis durch, dass allein durch die Berechnung und verursachungsgemäße Verrechnung der betrieblichen Umweltschutzkosten nur selten Einsparungsmöglichkeiten aufgezeigt werden (2). Um dies $\mathrm{zu}$ erreichen, müssen insbesondere im Bereich der Abfälle, Abwässer und Ausschüsse sämtliche von diesen Stoffflüssen verursachten Kosten berücksichtigt werden. Häufig überschreiten die Materialkosten und die Kosten, die im Vorfeld in der 
Produktion entstehen, also bevor der jeweilige Stoff als Abfall oder sonstiger Reststoff anfällt, deutlich die Umweltschutzkosten der internen und externen Entsorgungsprozesse. Diese Kostenstruktur, die weder von der klassischen Kostenrechnung noch von der Umweltschutzkostenrechnung angemessen abgebildet wird, berücksichtigt die Flusskostenrechnung systematisch, indem die Stofffliusse als Kostentreiber interpretiert werden. Das Instrument Flusskostenrechnung kann folgende Funktionen erfüllen:

- Identifizierung von Kostensenkungspotenzialen: Mit der Erstellung der Nebenrechnung ist eine detaillierte Betrachtung der einzelnen Prozesse und die Berechnung der entstehenden Flusskosten fuir die anfallenden Abfall- und Ausschussmengen erforderlich, so dass bereits in diesem Arbeitsschritt erste Kostensenkungspotenziale identifiziert werden.

- Identifizierung von Schwachstellen in der Kostenrechnung: Bei der Berechnung der Flusskosten werden implizit Plausibilitätstests der vorhandenen Daten und auf den zugrundeliegenden Berechnungen und Erfassungen durchgefïhrt. Weiterhin wird bei der Berechnung der Flusskosten deutlich, dass bestimmte Kostenblöcke, insbesondere die Flusskosten für Abfälle, Ausschuss und Verpackungen, nicht angemessen in den Berichten des Rechnungswesens abgebildet werden.

- Unterstützung des kontinuierlichen Verbesserungsprozesses: Die Entwicklung der Flusskosten von Abfällen, Emissionen, Verpackungen und anderen umweltrelevanten Stoffflüssen wird aufgezeigt und nachvollziehbar gemacht, so dass diese Stoffflüsse besser als bisher im Rahmen der Kostenplanung und Kostenkontrolle berücksichtigt werden.

- Auslösung eines Lermprozesses bei einer vergleichsweise großen Anzabl von Mitarbeitern: Durch die Flusskostenrechnung werden neue Perspektiven auf die Zusammensetzung der Herstellkosten gewonnen.

- Verbesserung der Informationslage für die Produktentwicklung und die Investitionsrechnung: Die neue Kostenperspektive verdeutlicht die Eigenschaft der betrieblichen Stoffflüsse als Kostentreiber.

Der im Vergleich zu Umweltkennzahlen höhere Aufwand bei der Einführung der Flusskostenrechnung wird mit einem deutlich breiteren Nutzungsspektrum aufgewogen.

\section{- Aufschlussreicher Vergleich}

Die zwei betrachteten Instrumente lassen sich unter anderem hinsichtlich der vorgestellten Funktionen, die sie erfüllen bzw. unterstiitzen, und beziiglich verschiedener Vor- und Nachteile unterscheiden (vgl. Tab. 1).

Der Vergleich der Funktionen macht zunächst deutlich, dass beide Instrumente sowohl die Ermittlung von Optimierungspotenzialen als auch einen institutionalisierten kontinuierlichen Verbesserungsprozess mit Zielvorgaben und zeitnahen Erfolgskontrollen unterstiitzen und hier gegenüber Umweltbilanzen deutliche Vorteile aufweisen.

Wie erfolgreich Umweltkennzahlen und die Flusskostenrechnung eine ökologisch ökonomische Optimierung der Produktion vorantreiben kön- nen, zeigen Erfahrungen aus Forschungsprojekten des IÖW. Drei mittelständische Unternehmen mit bestehenden und engagiert praktizierten Umweltmanagementsystemen samt Umweltbilanzen konnten bei der Einführung von Umweltkennzahlen noch wichtige Verbesserungsmöglichkeiten aufdecken und insbesondere neue Ziele fuir den kontinuierlichen Verbesserungsprozess formulieren. Mit der Flusskostenrechnung würde man in diesen Unternehmen unseres Erachtens keine weiteren Effizienzpotenziale aufdecken können.

Betrachtet man umgekehrt Unternehmen, die in Ergänzung ihres Umweltmanagementsystems die Flusskostenrechnung eingefuihrt haben, dann wurden auch hier bedeutende Optimierungspotenziale identifiziert (3). Auch in diesen Unternehmen ist davon auszugehen, dass mit Hilfe anderer Instrumente keine zusätzlichen ökologischen Effizienzpotenziale aufgedeckt werden können. Allerdings muss hier berücksichtigt werden, dass mit der Einfuihrung der Flusskostenrechnung nicht nur die Datenlage in der Kostenrechnung sondern auch auf der Mengenebene deutlich verbessert wurde und in den meisten Fällen ergänzend auch Umweltkennzahlen eingefuihrt wurden. Denn in mehreren Bereichen, wie z.B. der Energiewirtschaft, sind physikalische Größen für Steuerungs- und Überwachungszwecke deutlich besser geeignet als Kostenwerte.

Die Flusskostenrechnung wie auch Umweltkennzahlen wurden bislang in Unternehmen mit bestehenden Umweltmanagementsystemen eingeführt. Für diese Unternehmen gehört die Erstellung der Umweltbilanz zum guten

\begin{tabular}{|c|c|c|c|}
\hline Instrument & Funktionen & Vorteile & Nachteile \\
\hline $\begin{array}{l}\text { Umwelt- } \\
\text { kenmzahlen }\end{array}$ & $\begin{array}{l}\text { - Feststellung von Trends } \\
\text { - Schwachstellenanalyse } \\
\text { - Unterstüzzung des KVP über } \\
\text { Zielsetzung und Zielverfolgung } \\
\text { - Benchmarking (innerbetrieblich } \\
\text { und betriebsubergreifend) }\end{array}$ & 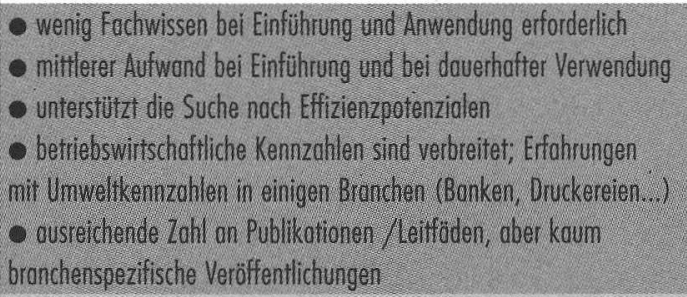 & $\begin{array}{l}\text { - je nech Branche noch wenig } \\
\text { Effahrungen zur internen Verwendung } \\
\text { Kostenbedeutung der Stoffströme } \\
\text { wird nicht ersichtlich } \\
\text { - Konn ds, Oko-instrument" interpre- } \\
\text { tiert werden } \rightarrow \text { Akzeptonzprobleme }\end{array}$ \\
\hline $\begin{array}{l}\text { Fluss-: } \\
\text { kosten- } \\
\text { rechnung }\end{array}$ & $\begin{array}{l}\text { - Schwachstellenanalyse, Identifikation } \\
\text { von Kostensenkungspotenzialen. } \\
\text { - Aufdeckung von Schwachstellen } \\
\text { in der Kostenrechnung } \\
\text { - Feststellung von Trends } \\
\text { - Unterstützung des KVP } \\
\text { - Auslösung innerbetrieblicher Lernprozesse } \\
\text { - Verbesserung der Informationslage } \\
\text { für F\&E und Investitionsrechnung }\end{array}$ & $\begin{array}{l}\text { - Unterstützt die Suche nach Effizienzpotenzialen systematisch } \\
\text { - Kostenbedeutung der Stoffströme wird ersichtlich } \\
\text { - "Kosten-Instrument" findet einfach Anerkennung im Unternehmen }\end{array}$ & $\begin{array}{l}\text { - Fochwissen bei Einführung und } \\
\text { Anwendung erforderlich } \\
\text { (derzeit) hoher Aufwond bei } \\
\text { daverhafter Integration } \\
\text { Instrument ist noch wenig verbreitet } \\
\text { und erst allmählich ousgereift } \\
\text { geringe Zahl an Publikationen/ } \\
\text { Leifföden; keine branchenspezifischen } \\
\text { Veröffentlichungen }\end{array}$ \\
\hline
\end{tabular}

Quelle: eigene Darstellung 
Umweltmanagement. Häufig wird bei der Einfuihrung von Umweltmanagementsystemen vorgeschlagen, jährlich eine aktuelle Umweltbilanz zu erstellen. Wenn bereits mit Kennzahlen oder Kostengrößen die Entwicklung der wesentlichen umweltrelevanten Stoff- und Energieströme verfolgt wird, dürfte es jedoch ausreichen, die vollständige Umweltbilanz in größeren Abständen, z.B. nur alle drei Jahre, zu erstellen.

Betrachtet man die vielfältigen Funktionen der Instrumente, so ist festzustellen, dass ein Unternehmen durch die Anwendung beider Instrumente einen erheblichen Nutzen für das betriebliche Umweltmanagement erzielen kann, wobei beide jeweils spezifische Vor- und Nachteile aufweisen (vgl. Tabelle 1). Dieser Nutzen wirkt sich vor allem auf die zentrale Aufgabe der systematischen Planung und Steuerung des kontinuierlichen Verbesserungsprozesses aus, denn durch die erhöhte Transparenz von Schwachstellen und Einsparpotenzialen im betrieblichen Umweltschutz können regelmäßig zielorientierte Maßnahmen abgeleitet und umgesetzt werden. Im Vergleich zur betrieblichen Umweltbilanz und zu Umweltkennzahlen wird durch die Anwendung der Flusskostenrechnung die weitgehendste Transparenz erreicht. Insbesondere für die Informationsversorgung der Produktentwicklung und für die Investitionsrechnung weist die Flusskostenrechnung daher wesentliche Stärken gegenuiber den anderen Instrumenten auf.

\section{- Praxisanwendung noch gering}

Dem erzielbaren Nutzen der beiden Instrumente steht eine verhältnismäßig geringe Anwendung in der Praxis des betrieblichen Umweltmanagements gegenüber. Dies ist auf zwei Ursachen zurückzuführen. Zum einen erfordert die Umsetzung der Flusskostenrechnung ein erhebliches Fachwissen im Bereich der betrieblichen Kostenrechnung, und es liegen für die Flusskostenrechnung wie auch Umweltkennzahlen noch keine umfassenden Praxiserfahrungen vor. Hier ist zu erwarten, dass durch eine weitere Praxiserprobung in entsprechenden Pilotprojekten die Anwenderfreundlichkeit der Instrumente erhöht werden kann. Zum anderen ist die Verfügbarkeit von Informationen $\mathrm{zu}$ betrieblichen Stoff- und Energieströmen als Grundlage für die Anwendung dieser Instrumente in den meisten Unternehmen noch sehr gering. Hier kann der effektive Einsatz von Betrieblichen Umweltinformationssystemen (BUIS) wesentlich zur Verbesserung der
Umsetzbarkeit der Instrumente beitragen. Diese Erfahrung hat sich innerhalb von Anwenderbefragungen (4) sowie zahlreichen Gesprächen mit Umweltmanagementpraktikern bestätigt.

In der derzeitigen Situation wird die Anwendung des Flusskostenansatzes „nur" in Unternehmen mit hohen Materialkosten und/oder hohen Entsorgungskosten relevant sein. Die anderen Unternehmen dürften daher bis zur Verbesserung der Softwareunterstiutzung die Anwendung von Umweltkennzahlen bevorzugen. Aber auch hier muss die - in der Fachöffentlichkeit sicherlich schon profan klingende - Erkenntnis aus der Entwicklung der Flusskostenrechnung angemessen berïcksichtigt werden: Umweltschutzkosten sind nur die Spitze des Eisberges, die größten Kosten des Abfalls (und anderer Emissionen) liegen in der Beschaffung und Verarbeitung der Materialien.

\section{Anmerkungen}

(1) Vgl. Kottmann, H./ T. Loew/ J. Clausen: Umweltmanagement mit Kennzahlen, Vahlen, München 1999.

(2) Vgl. z.B. Loew, I./ K. Fichter: Umweltkostenmanagement auf neven Wegen, in: Ökologisches Wirtschaften 2/1998, S. 28-30.

(3) Vgl. Ministerium für Wirtschaft, Verkehr und Landesentwicklung (Hrsg.): Leiffaden Flusskostenrechnung - Kostensenkung und Umweltentlastung durch eine Materialflussorientierung in der Kostenrechnung, Wiesbaden 1999 (im Erscheinen), S. $16 \mathrm{ff}$.

(4) Vgl. Rey, U./ G. Jürgens/ A. Weller: Betriebliche Umweltinformationssysteme - Anforderungen und Einsatz, Ergebnisse einer Befragung von Anwendern und Anbietern von informationstechnischen Unterstützungssystemen im Umweltmanagement, Fraunhofer IRB-Verlag, Stuttgart 1998.

(5) Vgl. Bullinger, H./ G. Jürgens/ U. Rey (Hrsg.): Betriebliche Umweltinformationssysteme in der Praxis, Fraunhofer IRB-Verlag, Stuttgart 1999.

Die Autoren
Thomas Loew ist wissenschaftlicher Mitarbeiter im
IÖW-forschungsfeld Ökalogische Unternehmenspolitik.
Kontakt: IÖW, Potsdamer Str. 105, 10785 Berlin,
E-mail: Thomas.Loew@ioew.de
Gunnar Jürgens ist wissenschaftlicher Mitarbeiter im
Fraunhofer Institut für Arbeitswirtschaft und Organi-
sation IIAO).
Kontakr: Fraunhofer IAO, Nobelstr. 12,
70569 Stuttgart,
E-mail: gunnar.juergens@iao.fhg.de

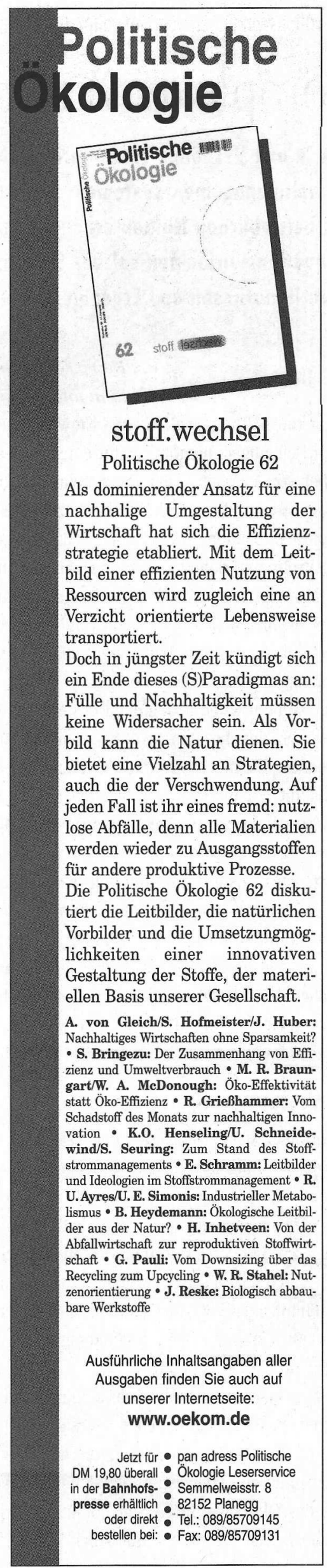


(c) 20I0 Authors; licensee IÖW and oekom verlag. This is an article distributed under the terms of the Creative Commons Attribution Non-Commercial No Derivates License (http://creativecommons.org/licenses/by-nc-nd/3.o/), which permits unrestricted use, distribution, and reproduction in any medium, provided the original work is properly cited. 